\title{
van der Waals interactions mediating the cohesion of fullerenes on graphene
}

\author{
M. Švec, ${ }^{1, *}$ P. Merino, ${ }^{2}$ Y. J. Dappe, ${ }^{3,4}$ C. González, ${ }^{1}$ E. Abad, ${ }^{5,6}$ P. Jelínek, ${ }^{1}$ and J. A. Martín-Gago ${ }^{2,7}$ \\ ${ }^{1}$ Institute of Physics, Academy of Sciences of the Czech Republic, Cukrovarnicka 10, CZ-16200 Prague, Czech Republic \\ ${ }^{2}$ Centro de Astrobiología INTA-CSIC, Carretera de Ajalvir, km. 4, ES-28850 Madrid, Spain \\ ${ }^{3}$ CEA, IRAMIS, SPCSI, F-91191 Gif sur Yvette, FRANCE \\ ${ }^{4}$ Service de Physique de l'Etat Condensé, DSMIIRAMIS/SPEC, CEA Saclay URA CNRS 2464, F-91191 Gif-Sur-Yvette Cedex, France \\ ${ }^{5}$ Departamento de Física Teórica de la Materia Condensada, Universidad Autónoma de Madrid, ES-28049 Madrid, Spain \\ ${ }^{6}$ Computational Biochemistry Group, Institute of Theoretical Chemistry, University of Stuttgart, Pfaffenwaldring 55 , \\ D-70569 Stuttgart, Germany \\ ${ }^{7}$ Instituto de Ciencia de Materiales de Madrid (CSIC), c/Sor Juana Inés de la Cruz, ES-28049 Madrid, Spain
}

(Received 22 May 2012; revised manuscript received 4 September 2012; published 24 September 2012)

Fullerenes on single-layer epitaxial graphene are a model system to study very faint interactions at a molecular level. By a variable temperature scanning tunneling microscope we have been able to study ordered fullerene layers at $40 \mathrm{~K}$, exclusively bound by van der Waals interactions. The experimentally determined adsorption geometry of the molecules is computationally confirmed only if van der Waals interactions are included in the calculation formalism. The relative orientation of fullerenes in their close-packed arrangement is found to be the crucial factor for determining the total energy. Observation of collective movements of fullerene islands points out the weak coupling to the substrate and the important role of the van der Waals cohesion forces within.

DOI: 10.1103/PhysRevB.86.121407

PACS number(s): 81.05.ub, 81.05.ue, 36.40.Sx, 37.10.Mn

Mechanical stability, friction, or adhesion are among the physical properties that strongly depend on van der Waals (vdW) interactions. This is also true for the nanoscale. The nucleation and growth of molecular surface structures involve dynamic processes such as diffusion, molecular rotations, or conformational changes, which rely also on vdW intermolecular interactions. ${ }^{1,2}$ Moreover, self-assembly and adsorption studies focus on determining the preferred adsorption site and configuration, the adsorbate-adsorbate interaction, and the distance between the adsorbate and substrate (e.g., Ref. 3). There is an increasing interest in the role of $\mathrm{vdW}$ interactions of organic molecules on graphite and other surfaces. ${ }^{4,5}$

Generally, long-distance forces as vdW are not described by the most widely used functionals of the density-functional theory (DFT). Thus, in many of the works performed until now they are simply not included. However, when planar systems of carbon-based materials are in question, they require a different approach to the interplay between intermolecular (lateral) and adsorbate-substrate (vertical) interactions in determining the properties of ordered molecular structures. To evidence the important role of the $\mathrm{vdW}$ interactions in adsorption processes we have chosen a system of a very weakly interacting substrate and adsorbate, single-layer graphene (SLG) ${ }^{6}$ and fullerenes $\left(\mathrm{C}_{60}\right){ }^{7}$ The fact that both materials consist exclusively of carbon atoms arranged in an atomically thin planar mesh without $\mathrm{H}$ or any other atoms inside the atomic structure that could lead to long-range $\mathrm{H}$-bond interactions makes this system a good prototype for a demonstration of the effect of these forces at a molecular level.

Thus, we consider the $\mathrm{C}_{60}$ on SLG grown on $6 \mathrm{H}-$ $\mathrm{SiC}(0001)^{8-10}$ as a model system to test the strength of the $\mathrm{vdW}$ forces and mutual interactions that occur between neutral inert nanostructures. $\mathrm{C}_{60}$ adsorbed on surfaces generally tend to form hexagonal close-packed arrangements ${ }^{11}$ in order to optimize their lateral interactions. In very recent studies of $\mathrm{C}_{60}$ molecules deposited on SLG epitaxially grown on metal, ${ }^{12,13}$ it has been shown that the interaction between the molecules and the substrate, and consequently the molecular arrangement, is ruled by the moiré unit cell. The $\mathrm{C}_{60}$ coming to the surface are trapped in potential wells of the moiré valleys where the substrate SLG is more reactive, thus forming pinning centers for the other molecules that arrange in between. In contrast to these studies, we found a much weaker interaction of the $\mathrm{C}_{60}$ with the SLG grown on $6 \mathrm{H}-\mathrm{SiC}(0001)$, which lead us to a workbench to discuss the origin of the bonding mechanism in weakly interacting systems. Very recently a study of a very similar system appeared, ${ }^{14}$ which characterizes the basic behavior of the $\mathrm{C}_{60}$ on a graphene layer at $6 \mathrm{H}-\mathrm{SiC}(0001)$ near to one monolayer coverage and studies its electronic properties based on scanning tunneling spectroscopy.

We employed a variable temperature scanning tunneling microscope (VT-STM) and DFT-vdW calculations ${ }^{15-17}$ to prove that this system is solely governed by vdW forces. We determined the adsorption geometry of the molecules and we observed their collective motion. We also show that including $\mathrm{vdW}$ contribution in the calculations is necessary in order to fully describe theoretically the interaction between the $s p^{2}$ systems.

STM images of submonolayer coverage of $\mathrm{C}_{60}$ molecules deposited at room temperature (RT) onto a $6 \mathrm{H}-\mathrm{SiC}(0001)$ substrate partly covered with SLG show slightly disordered close-packed hexagonal planar islands of $\mathrm{C}_{60}$ exclusively on the $\mathrm{SiC}$ buffer layer which has a quasi- $6 \times 6$ periodicity (hereafter referred to as $6 \times 6$ ). ${ }^{9}$ That is, neither islands nor single molecules were spotted on the areas covered by SLG, because adsorbed $\mathrm{C}_{60}$ diffuse out of the SLG regions. ${ }^{18}$ On the contrary, when we evaporate $\mathrm{C}_{60}$ on the sample kept at a low temperature of $40 \mathrm{~K}$ (LT), STM images show the formation of $\mathrm{C}_{60}$ epitaxial structures on SLG in the form of well-ordered planar islands with a configuration that seems to have a twofold symmetry. Figures 1(a) and 1(b) show the STM topography of islands on both types of surfaces and adsorbed 

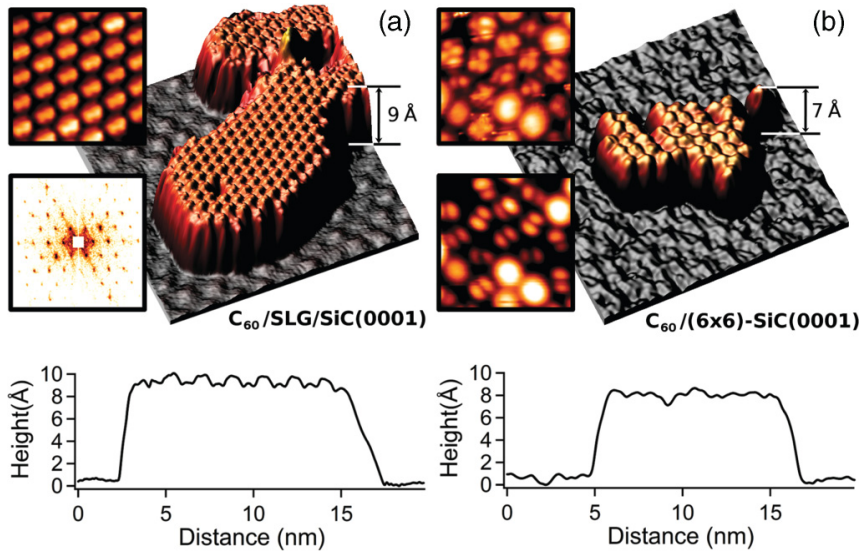

FIG. 1. (Color online) Three-dimensional (3D) representation of $20 \times 20 \mathrm{~nm}^{2}$ empty states STM topography on $\mathrm{C}_{60}$ islands and their corresponding profiles: (a) SLG taken at $600 \mathrm{mV}, 100 \mathrm{pA}$, with a $5 \times 5 \mathrm{~nm}^{2}$ detail and FFT power spectrum of the entire area, showing a clear $4 \times 4$ pattern of the close-packed arrangement of the molecules on SLG. (b) $(6 \times 6)-\mathrm{SiC}(0001)$ recorded at $1000 \mathrm{mV}, 100 \mathrm{pA}$, and $5 \times$ $5 \mathrm{~nm}^{2}$ insets showing two different types of submolecular resolution. Both images were obtained at $40 \mathrm{~K}$.

molecules in detail, the latter imaged at two different levels of resolution.

At first glance the properties of the $\mathrm{C}_{60}$ islands on $6 \times 6$ [Fig. 1(b)] are very similar to the ones reported before at RT. ${ }^{19,20}$ The molecules inside the island are present in various orientations, i.e., different molecular orbitals (MO) are exposed to the probe during scanning. Their corrugation is $0.45 \AA \mathrm{rms}$ and the base apparent height is $7.2( \pm 0.5) \AA$. On larger scales, two quasiperiodic arrangements could be found corresponding to a pair of twin domains appearing at $\pm 20^{\circ}$ (with $1^{\circ}$ error) with respect to $6 \times 6$.

On the other hand, the $\mathrm{C}_{60}$ islands on SLG in Fig. 1(a) show lower corrugation $(0.28 \AA \mathrm{rms})$, a considerably denser packing, and an almost perfect order. According to the FFT power spectrum of the STM image in the inset of Fig. 1(a), the $\mathrm{C}_{60}$ molecules arrange in a $4 \times 4$ commensurate superstructure with respect to the SLG lattice. As a rule, the islands are hexagonally shaped with edge angles of $120^{\circ}$, which is an expected kind of behavior, since it has been already observed in the first layer of $\mathrm{C}_{60}$ on highly oriented pyrolitic graphite. ${ }^{21}$ The profile analysis of the islands gives an apparent height of $8.8( \pm 0.3) \AA$ and interestingly does not show medium-scale corrugation that could be expected due to the underlying $6 \times 6$ superperiodicity (resulting in a quasi- $6 \times 6$ corrugation of the SLG in the STM topography ${ }^{9}$ ). These findings are consistent with the recent observation made by Cho et al.. ${ }^{14}$

The detail in the inset of Fig. 1(a) shows submolecular resolution, which suggests the adsorbed molecules on SLG are all equally oriented. The submolecular structure of each $\mathrm{C}_{60}$ consists of two bright lobes that shall correspond to their MO, which may be considered as a fingerprint for their orientation. ${ }^{11,22,23}$ The most intense features are usually assigned to the $\mathrm{C}_{60}$ pentagons. However, in our case, the MO contrasts are slightly varying between subsequent STM images while maintaining the overall character-see Fig. 2. That evidences changes of the probe itself and prevents us from

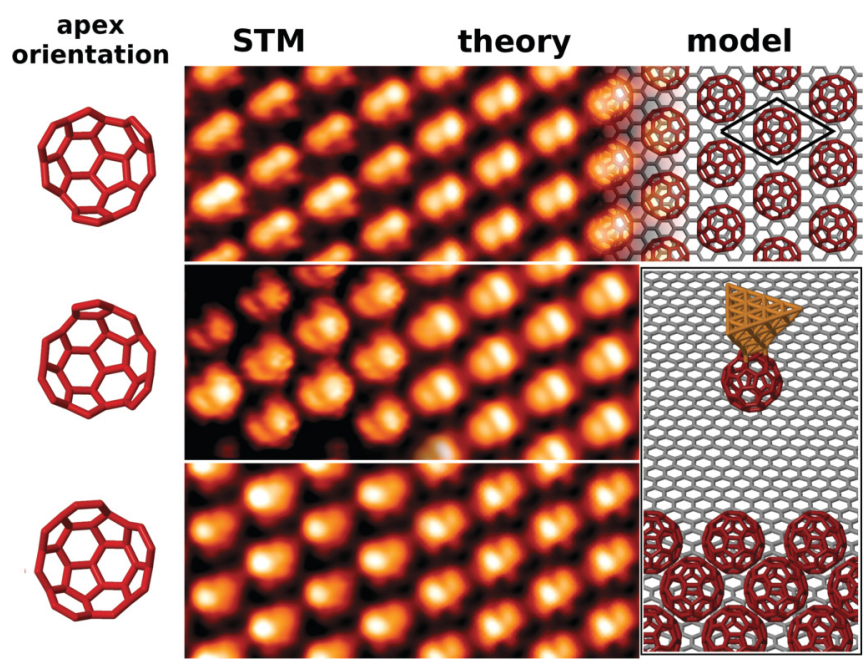

FIG. 2. (Color online) STM topography obtained at $600 \mathrm{mV}$ bias voltage on the $4 \times 4 \mathrm{C}_{60}$ on SLG with varying contrast. The experimental images are compared to the uniquely matching theoretical simulation of STM topography using a $\mathrm{C}_{60}$ adsorbed on a metallic tip apex in various orientations, scanned above the model structure of $4 \times 4 \mathrm{C}_{60}$ on SLG. The lower halves of the probe $\mathrm{C}_{60}$ are schematically shown near to the appropriate images. The 3D scheme in the inset illustrates the situation.

directly relating the observed contrast to any expected MO of $\mathrm{C}_{60}$ molecules previously reported. ${ }^{11,22,23}$ To unambiguously determine the correct orientation of the molecules on the surface based on these data, we have to assume a $\mathrm{C}_{60}$ being picked up by the reactive metallic tip apex before the acquisition of the images, since this process is commonplace, especially in LT STM sessions-e.g. Refs. 24 and 25. Therefore, the observed STM contrast on the MO is most likely influenced by imaging of a $\mathrm{C}_{60}$ by another $\mathrm{C}_{60}$ adsorbed on the tip apex.

Consequently, the most important questions that arise from the experiment are about (i) the detailed role of the $\mathrm{vdW}$ in the well-ordered $4 \times 4 \mathrm{C}_{60} / \mathrm{SLG}$ system and (ii) the orientation of the $\mathrm{C}_{60}$ with respect to the SLG lattice.

We performed extensive DFT-vdW calculations in order to understand the role of the vdW in stabilizing this structure. Several groups have developed DFT-based calculations including the vdW interaction. ${ }^{15,26-29}$ Here we use the LCAO- $S^{2}$ previously applied to SLG and graphene-like materials. ${ }^{15,16}$ In this formalism, we consider two contributions. The first arises from the small overlaps between the electronic wave functions of the $\mathrm{C}_{60}$ and the SLG, leading to an electronic repulsion, and the second, which is the vdW interaction itself, is due to oscillating dipoles in both interacting systems. These two contributions are treated in perturbation theory from a DFT calculation using the FIREBALL code. ${ }^{17,30-32}$ This method takes into account particularly the $\pi-\pi$ interactions since the corresponding overlaps are the dominant effect in this weakly interacting system. The underlying SiC buffer ${ }^{33-37}$ layer was neglected, since the expected energy contribution to the $\mathrm{C}_{60}$ total energy due to the vdW interaction is at least an order of magnitude lower than the contribution due to the presence of SLG, ${ }^{18}$ considering the large separation of SLG and the buffer layer. ${ }^{38}$

In the calculations we used more than 20 different adsorption geometries of $\mathrm{C}_{60} / \mathrm{SLG}$ in a $4 \times 4$ periodicity. ${ }^{18}$ 
TABLE I. Total energy values per $\mathrm{C}_{60}$ for their various orientations in a $4 \times 4$ SLG supercell, with and without considering vdW interactions ( $E_{\mathrm{vdW}}$ and $E_{\mathrm{DFT}}$ ) and the associated equilibrium distances $d_{\mathrm{vdW}}$ and $d_{\mathrm{DFT}}$. $E_{\mathrm{vdW}}$ is a sum of two contributions: the cohesion energy between the $\mathrm{C}_{60}$ molecules, $E_{\mathrm{C} 60-\mathrm{C} 60}$, and the interaction energy between $\mathrm{C}_{60}$ and the SLG substrate, $E_{\mathrm{C} 60-\mathrm{SLG}}$. The structure with the lowest energy value is taken as a reference for calculation of the relative total energy $\Delta E_{\mathrm{vdW}}$.

\begin{tabular}{lcccc}
\hline \hline $\begin{array}{l}\mathrm{C}_{60} \text { orientation } \\
\text { SLG adsorption site }\end{array}$ & $\begin{array}{c}6: 6 / 30^{\circ} \\
\text { Hollow }\end{array}$ & $\begin{array}{c}6: 6 / 0^{\circ} \\
\text { Hollow }\end{array}$ & $\begin{array}{c}\text { Hex } / 0^{\circ} \\
\text { Adatom }\end{array}$ & $\begin{array}{c}\text { Hex } / 0^{\circ} \\
\text { Dimer }\end{array}$ \\
\hline$d_{\mathrm{DFT}}(\AA)$ & 3.4 & 3.3 & 3.5 & 3.6 \\
$E_{\mathrm{DFT}}(\mathrm{eV})$ & -0.006 & -0.006 & -0.004 & -0.004 \\
$d_{\mathrm{vdW}}(\AA)$ & 2.7 & 2.7 & 2.9 & 2.9 \\
$E_{\mathrm{C} 60-\mathrm{C} 60}(\mathrm{eV})$ & -0.880 & -0.871 & -0.680 & -0.645 \\
$E_{\mathrm{C} 60-\mathrm{SLG}}(\mathrm{eV})$ & -1.007 & -1.010 & -1.019 & -1.016 \\
$E_{\mathrm{vdW}}(\mathrm{eV})$ & -1.887 & -1.881 & -1.696 & -1.659 \\
$\Delta E_{\mathrm{vdW}}(\mathrm{eV})$ & 0.000 & +0.006 & +0.189 & +0.226 \\
\hline \hline
\end{tabular}

These calculations have confirmed that the energetically most favorable structures are indeed those with a high symmetry. To discriminate the contribution of the $\mathrm{vdW}$ forces in the global structure, we made DFT calculations with and without incorporation of the vdW forces. The most stable structures among all the probed adsorption sites and molecular orientations are presented in Table I. The molecular orientations are labeled by $\mathrm{a} \mathrm{C}_{60}$ feature exposed to the surface and the angle of rotation around the $z$ axis (perpendicular to the surface plane) with respect to the diagonal of a $1 \times 1$ unit cell of SLG. Thus, the $6: 6 / 0^{\circ}$ orientation corresponds to an adsorption of a $\mathrm{C}_{60}$ by a dimer shared between two adjacent hexagons parallel to a C-C bond in SLG; the $6: 6 / 30^{\circ}$ is identical to the $6: 6 / 0^{\circ}$ rotated by $30^{\circ}$ around the $z$ axis; hex $/ 0^{\circ}$ is a hexagon aligned with the SLG hexagons, etc. ${ }^{18}$ There are three possible adsorption sites of high symmetry on the SLG: on top of a C atom (adatom), in the center of a hexagon (hollow), and above the center of a C-C bond (dimer).

In Table I we see that the lowest total energy $\left(E_{\mathrm{vdW}}\right)$ structure calculated including the $\mathrm{vdW}$ interaction is the $6: 6 / 30^{\circ}$ in a hollow site of SLG, which is used as the reference value for the relative energy of adsorption $\left(\Delta E_{\mathrm{vdW}}\right)$. The closest structure in terms of energy is the $6: 6 / 0^{\circ}$, also in a hollow site, with a total energy higher by $6.44 \mathrm{meV} / \mathrm{C}_{60}$. Taking into account that the experiments were performed at $40 \mathrm{~K}$, the thermal energy is about $3 \mathrm{meV}$, which is approximately half of the difference between the two most favorable structures. Consequently, the system at $40 \mathrm{~K}$ should prefer the hollow $6: 6 / 30^{\circ}$ adsorption geometry over the hollow $6: 6 / 0^{\circ}$. The rest of the structures present values of the total energy that are much larger, indicating the strong influence of the $\mathrm{C}_{60}$ orientation with respect to each other in the value on the vdW interaction. Remarkably, the main difference in the total energy comes from the molecule-molecule interactions $\left(E_{\mathrm{C} 60-\mathrm{C} 60}\right)$ rather than from the interaction with the surface $\left(E_{\mathrm{C} 60-\mathrm{SLG}}\right)$, which is only slightly modified when the molecule is placed with a different orientation. As the $E_{\mathrm{C} 60-\mathrm{C} 60}$ differs for each adsorption orientation, it has the ultimate role in the final value of the $E_{\mathrm{vdw}}$. Charge transfer from the surface to a $_{60}$ is negligible, amounting to $\approx 0.03$ electrons $/ \mathrm{C}_{60}$, which has been suggested recently. ${ }^{14}$
Interestingly, when we perform the total energy calculations without the vdW interactions $\left(E_{\mathrm{DFT}}\right)$, all four structures result about the same energy (with differences less than $3 \mathrm{meV} / \mathrm{C}_{60}$ ) and therefore the orientation of the molecules in the islands on the surface would not have any particular preference under our experimental conditions. The introduction of the $\mathrm{vdW}$ interaction results in a considerable reduction of the $\mathrm{C}_{60}$-SLG distance $\left(d_{\mathrm{vdW}}\right.$ compared to $\left.d_{\mathrm{DFT}}\right)$. That is a clear indication that the $\mathrm{vdW}$ interactions cannot be neglected in any similar system.

Aware that the total energy difference between $6: 6 / 30^{\circ}$ and 6:6: $/ 0^{\circ}$ is very small, we performed STM simulations for these adsorption geometries to elucidate which is the structure observed experimentally. ${ }^{39}$ A set of 60 tips consisting of a pyramid of 35 metal atoms and a $\mathrm{C}_{60}$ molecule attached to the apex in various geometries has been used ${ }^{40}$ as a probe over both candidates. The calculated images were carefully compared to the experimental images with varying contrast. We found the agreement only for the structures based on the $6: 6 / 30^{\circ}$ orientation, as in the example in Fig. 2, where the best agreement is obtained for the $6: 6 / 30^{\circ}$ imaged with appropriate orientations of the $\mathrm{C}_{60}$ on the tip apex. The couples of bright lobes in the images systematically correspond to a pair of pentagons linked by a dimer between two hexagons, which is crucial to decide the mutual orientation of the molecules in the $4 \times 4$ structure. The calculated images are all similar to an image produced by a simple metallic tip, somewhat modified by the effect of the $\mathrm{MO}$ of a rotated $\mathrm{C}_{60}$ on the tip. Considering the high probability of having a $\mathrm{C}_{60}$ on the tip apex leads us to inevitably conclude that any spectroscopic information obtained by scanning tunneling spectroscopy on this system can be significantly distorted. In particular, the width of the gap between the highest occupied and lowest unoccupied states of a $\mathrm{C}_{60}$ is likely to be overestimated. ${ }^{14}$

So far, we have pointed out the dominance of $\mathrm{vdW}$ intermolecular attraction between the $\mathrm{C}_{60}$ molecules on SLG. Due to a distinct decay and strength of this force one can expect a qualitatively different dynamical behavior of the molecules in this system. Movement of a single molecule away from the islands is highly unfavorable, because it has to overcome the energy barrier created by the $\mathrm{vdW}$ interactions with the nearest neighbors, but it will likely occur along the edge of an island. We observed such a process and it was enhanced by interactions with the scanning tip. ${ }^{18}$ We successfully attempted to observe $\mathrm{C}_{60}$ diffusion in an experiment by means of fast and reiterate scanning of the same region. Surprisingly, the islands that were not pinned by any defects (step edge, impurity, etc.) revealed a much faster mode of mass transport. Figure 3 shows a sequence of tip-induced changes undergone by an island consisting of $50 \mathrm{C}_{60}$ molecules.

The observed area contains two pinned and thus rather stable islands A and C, plus a free and a very mobile island B which is apparently directed by the sense of the scanning, i.e., alternating upwards and downwards. Importantly, the shape of $\mathrm{A}$ and $\mathrm{C}$ does not vary strongly from one image to the next. That means the $\mathrm{C}_{60}$ migration along the island edges is slow and not many events are missed. A consequent interpretation of the movement of B is only possible by collective motion, as seen in the images between the time stamps 2:48 and 8:24, where both the shape of the island and the number of its molecules remain preserved. Island A serves only as a pivot 


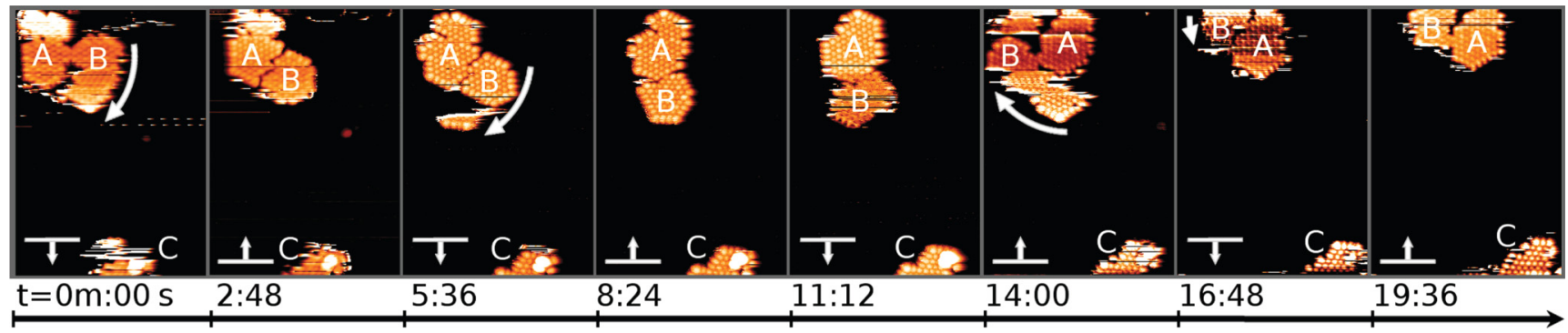

FIG. 3. (Color online) Sequence of STM topographic images taken at $1500 \mathrm{mV}$ and $100 \mathrm{pA}$ on SLG with three islands of $\mathrm{C}_{60}$. Islands A and $\mathrm{C}$ are pinned by a step edge and a surface defect, respectively. The whole island B is undergoing movements (marked by curved arrows) that apparently conserve the overall shape of the island between 2:48 and 8:24. The movements of the island are apparently correlated with the direction of scanning (denoted by vertical arrows with horizontal bars).

point as B is changing its orientation with the substrate by $30^{\circ}$. By such rotation of the island, the unit cell is temporarily changed to $(\sqrt{3} \times \sqrt{3}) R 30^{\circ} \mathrm{C}_{60}$ coincident with a $7 \times 7$ SLG, where the density of the molecules in the layer is changed only by $2 \%$ (one molecule per $4 \times 4$ to three molecules per $7 \times 7$ ). This observation is fully consistent with the balance between the adsorption and cohesion energies predicted by the theory. A diffusion barrier of $\mathrm{C} \mathrm{C}_{60} / \mathrm{SLG}$ is inherently very low and the attractive force between the $\mathrm{C}_{60}$ caused by the vdW interactions can reach over distances of several SLG unit cells. Consequently, in the absence of a strong site-specific bonding mechanism, the cohesion force between $\mathrm{C}_{60}$ molecules is the crucial factor in the stability of the islands.

In conclusion, the $\mathrm{C}_{60}$ /SLG behaves as a prototype of a decoupled adsorbate system governed by $\mathrm{vdW}$ forces. Advanced formalism, including vdW forces, is necessary for a successful determination of the correct adsorption geometry of the molecules. Our results show that the orientation of the molecule within the structure plays a major role in the total energy evaluation. The collective movement of small molecular islands demonstrates the dominant role of the vdW interactions in this system and the decoupling of the $\mathrm{C}_{60}$ from the substrate. The agreement of the experimental observation with the total energy and STM image calculations indicates the need for including vdW to account for the weak interactions in $s p^{2}$ compounds.

P.M. acknowledges financial support from a Rafael Calvo Rodés scholarship. M.S. and P.J. acknowledge Grant No. GAAV IAA100100905 GACR, Project No. 204/10/0952. C.G. acknowledges the Jose Castillejo Grant from the Spanish Education Ministry and the CSIC JAE-DOC position.
*Corresponding author: svec@fzu.cz

${ }^{1}$ C. Stadler, S. Hansen, I. Kröger, C. Kumpf, and E. Umbach, Nat. Phys. 5, 153 (2009).

${ }^{2}$ P. N. Dickerson, A. M. Hibberd, N. Oncel, and S. L. Bernasek, Langmuir 26, 18155 (2010).

${ }^{3}$ F. Hanke, S. Haq, R. Raval, and M. Persson, ACS Nano 5, 9093 (2011)

${ }^{4}$ A. Tkatchenko, L. Romaner, O. T. Hofmann, E. Zojer, C. AmbroschDraxl, and M. Scheffler, MRS Bull. 35, 435 (2010).

${ }^{5}$ V. G. Ruiz, W. Liu, E. Zojer, M. Scheffler, and A. Tkatchenko, Phys. Rev. Lett. 108, 146103 (2012)

${ }^{6}$ K. S. Novoselov, A. K. Geim, S. V. Morozov, D. Jiang, Y. Zhang, S. V. Dubonos, I. V. Grigorieva, and A. A. Firsov, Science 306, 666 (2004).

${ }^{7}$ H. W. Kroto, J. R. Heath, S. C. O'Brien, R. F. Curl, and R. E. Smalley, Nature (London) 318, 162 (1985).

${ }^{8}$ H. Shin, S. E. O’Donnell, P. Reinke, N. Ferralis, A. K. Schmid, H. I. Li, A. D. Novaco, L. W. Bruch, and R. D. Diehl, Phys. Rev. B 82, 235427 (2010).

${ }^{9}$ P. Martensson, F. Owman, and L. I. Johansson, Phys. Status Solidi B 202, 501 (1997).

${ }^{10}$ H. Huang, W. Chei, S. Chen, and A. T. S. Wee, ACS Nano 12, 2513 (2008).

${ }^{11}$ P. J. Moriarty, Surf. Sci. Rep. 65, 175 (2010).

${ }^{12}$ J. Lu, P. S. E. Yeo, Y. Zheng, Z. Yang, Q. Bao, C. K. Gan, and K. P. Loh, ACS Nano 6, 944 (2012).
${ }^{13}$ G. Li, H. T. Zhou, L. D. Pan, Y. Zhang, and J. H. Mao, Appl. Phys. Lett. 100, 013304 (2012).

${ }^{14}$ J. Cho, J. Smerdon, L. Gao, O. Süzer, J. R. Guest, and N. P. Guisinger, Nano Lett. 12, 3018 (2012).

${ }^{15}$ Y. J. Dappe, J. Ortega, and F. Flores, Phys. Rev. B 79, 165409 (2009).

${ }^{16}$ Y. J. Dappe, M. A. Basanta, F. Flores, and J. Ortega, Phys. Rev. B 74, 205434 (2006).

${ }^{17}$ J. P. Lewis, P. Jelínek, J. Ortega, A. A. Demkov, D. G. Trabada, B. Haycock, H. Wang, G. Adams, J. K. Tomfohr, E. Abad, H. Wang, and D. A. Drabold, Phys. Status Solidi B 248, 1989 (2011).

${ }^{18}$ See Supplemental Material at http://link.aps.org/supplemental/ 10.1103/PhysRevB.86.121407 for more information.

${ }^{19}$ W. Chen, H. L. Zhang, H. Xu, E. S. Tok, K. P. Loh, and A. T. S. Wee, J. Phys. Chem. B 110, 21873 (2006).

${ }^{20}$ W. Chen, S. Chen, H. L. Zhang, H. Xu, D. C. Qi, X. Y. Gao, K. P. Loh, and A. T. S. Wee, Surf. Sci. 610, 2994 (2007).

${ }^{21}$ H. Liu and P. Reinke, J. Chem. Phys. 124, 164707 (2006).

${ }^{22}$ X. Lu, M. Grobis, K. H. Khoo, S. G. Louie, and M. F. Crommie, Phys. Rev. B 70, 115418 (2004).

${ }^{23}$ G. Schull and R. Berndt, Phys. Rev. Lett. 99, 226105 (2007).

${ }^{24}$ G. Schull, T. Frederiksen, M. Brandbyge, and R. Berndt, Phys. Rev. Lett. 103, 206803 (2009).

${ }^{25}$ B. W. Heinrich, M. V. Rastei, D.-J. Choi, T. Frederiksen, and L. Limot, Phys. Rev. Lett. 107, 246801 (2011). 
${ }^{26}$ D. C. Langreth, M. Dion, H. Rydberg, E. Schröder, P. Hyldgaard, and B. I. Lundqvist, Int. J. Quantum Chem. 101, 599 (2005).

${ }^{27}$ A. Tkatchenko and M. Scheffler, Phys. Rev. Lett. 102, 073005 (2009).

${ }^{28}$ S. Lebegue, J. Harl, T. Gould, J. G. Angyan, G. Kresse, and J. F. Dobson, Phys. Rev. Lett. 105, 196401 (2010).

${ }^{29}$ G.-X. Zhang, A. Tkatchenko, J. Paier, H. Appel, and M. Scheffler, Phys. Rev. Lett. 107, 245501 (2011).

${ }^{30}$ J. P. Lewis, K. R. Glaesemann, G. A. Voth, J. Fritsch, A. A. Demkov, J. Ortega, and O. F. Sankey, Phys. Rev. B 64, 195103 (2001).

${ }^{31}$ P. Jelinek, H. Wang, J. P. Lewis, O. F. Sankey, and J. Ortega, Phys. Rev. B 71, 235101 (2005).

${ }^{32}$ M. A. Basanta, Y. J. Dappe, P. Jelinek, and J. Ortega, Comput. Mater. Sci. 39, 759 (2007).

${ }^{33}$ J. Hass, W. A. de Heer, and E. H. Conrad, J. Phys.: Condens. Matter 20, 323202 (2008).
${ }^{34}$ S. Kim, J. Ihm, H. J. Choi, and Y.-W. Son, Phys. Rev. Lett. 100, 176802 (2008).

${ }^{35}$ C. Riedl, C. Coletti, T. Iwasaki, A. A. Zakharov, and U. Starke, Phys. Rev. Lett. 103, 246804 (2009).

${ }^{36}$ J. Ristein, S. Mammadov, and T. Seyller, Phys. Rev. Lett. 108, 246104 (2012)

${ }^{37}$ C. Xia, S. Watcharinyanon, A. A. Zakharov, R. Yakimova, L. Hultman, L. I. Johansson, and C. Virojanadara, Phys. Rev. B 85, 045418 (2012).

${ }^{38}$ F. Varchon, R. Feng, J. Hass, X. Li, B. N. Nguyen, C. Naud, P. Mallet, J.-Y. Veuillen, C. Berger, E. H. Conrad, and L. Magaud, Phys. Rev. Lett. 99, 126805 (2007).

${ }^{39}$ J. M. Blanco, F. Flores, and R. Perez, Prog. Surf. Sci. 81, 403 (2006).

${ }^{40}$ G. Schull, Y. J. Dappe, C. Gonzlez, H. Bolou, and R. Berndt, Nano Lett. 11, 3142 (2011). 